\title{
Neural Dynamics during Resting State: A Functional Magnetic Resonance Imaging Exploration with Reduction and Visualization
}

\author{
Wei Li $\left(\mathbb{D},{ }^{1,2}\right.$ Miao Wang $\left(\mathbb{D},{ }^{1,2,3}\right.$ Wen Wen, ${ }^{1,2}$ Yue Huang, ${ }^{4}$ Xi Chen $\mathbb{D}^{1,},{ }^{1,2}$ Wenliang Fan $\left(\mathbb{D},{ }^{5}\right.$ \\ and Alzheimer's Disease Neuroimaging Initiative \\ ${ }^{1}$ School of Automation, Huazhong University of Science and Technology, Wuhan 430074, China \\ ${ }^{2}$ Image Processing and Intelligent Control Key Laboratory of Education Ministry of China, Wuhan 430074, China \\ ${ }^{3}$ China Ship Development and Design Center, Wuhan 430064, China \\ ${ }^{4}$ School of Electrical and Electronic Engineering, East China Jiaotong University, Nanchang 330013, China \\ ${ }^{5}$ Department of Radiology, Union Hospital, Tongji Medical College, Huazhong University of Science and Technology, \\ Wuhan 430022, China
}

Correspondence should be addressed to Wenliang Fan; 15827119065@163.com

Received 20 June 2017; Revised 19 December 2017; Accepted 11 January 2018; Published 19 June 2018

Academic Editor: Albert Diaz-Guilera

Copyright (C) 2018 Wei Li et al. This is an open access article distributed under the Creative Commons Attribution License, which permits unrestricted use, distribution, and reproduction in any medium, provided the original work is properly cited.

\begin{abstract}
The brain is a complex high-order system. Body movements or mental activities are both dependent on the transmission of information among billions of neurons. However, potential patterns are hardly discoverable due to the high dimensionality in neural signals. Previous studies have identified rotary trajectories in rhythm and nonrhythm movements when projecting the neural electrical signals into a two-dimensional space. However, it is unclear how well this analogy holds at the resting state. Given the low-frequency fluctuations noted during spontaneous neural activities using functional magnetic resonance imaging (fMRI), it is natural to hypothesize that the neural response at resting state also shows a periodic trajectory. In this study, we explored the potential patterns in resting state fMRI data at four frequency bands (slow 2-slow 5) on two cohorts, one of which consisted of young and elderly adults and the other of patients with Alzheimer's disease and normal controls (NC). The jPCA algorithm was applied to reduce the high-dimensional BOLD signal into a two-dimensional space for visualization of the trajectory. The results indicated that the "resting state" is a basic state showing an inherent dynamic pattern with a low frequency and long period during normal aging, with changes appearing in the rotary period at the slow 4 frequency band $(0.027-0.073 \mathrm{~Hz})$ during the pathological process of Alzheimer's disease (AD). These findings expand the original understanding that neural signals can rotate themselves and that motor executive signals consist of neural signals. Meanwhile, the rotary period at band slow 4 may be a physiological marker for $\mathrm{AD}$, and studies of this frequency band may be useful for understanding the potential pathophysiology of $\mathrm{AD}$ and ultimately facilitate characterization and auxiliary diagnosis of AD.
\end{abstract}

\section{Introduction}

The brain is a complex high-order system. Body movements or mental activities are all dependent on the transmission of information among billions of neurons [1]. Studies have generally suggested that neural activities characterize the response to a particular movement or task [2-5]. Therefore, scientists have been trying to decode the neural information to identify potential patterns, hoping to infer body movement or consciousness from neural signals [6-8].
Since 1995, functional magnetic resonance imaging (fMRI) has become an important technique to explore brain function because of its noninvasive and mature method of data acquisition [9]. In one approach, blood oxygenation level-dependent (BOLD) fMRI signals are recorded during imaging to measure the fluctuations of metabolism in different brain regions [10]. Early studies based on this approach generally focused on task-based assessments to identify the relationship between neural signals and task paradigms [11]. Recently, however, an increasing number of studies have 
started to investigate BOLD fluctuations at resting state to capture the intrinsic activities that could not be measured by task-based fMRI studies [11-13]. Previous studies have demonstrated the existence of low-frequency $(<0.1 \mathrm{~Hz})$ fluctuations in spontaneous neural activities $[14,15]$, which may be related to the basal metabolism and human physiological motions (such as respiration and cardiac pulsation) [1618]. Meanwhile, the default mode network (DMN), one of the resting state networks, was reported to be activated periodically as a result of periodic introspection [19]. These intrinsic activities or potential patterns are, naturally, buried in BOLD signals.

However, there are several challenges to extract the potential patterns from neural data. First, a lot of noise is mixed in the intrinsic fluctuations, complicating the process of decoding. Second, it is difficult to assess neural signals with high dimensionality. This lack of visualization impedes statistical analysis and observation. Potential patterns for the neuron populations are therefore overshadowed by tidal fluctuations of the complicated data. Finally, the analyses model may be complicated by the high dimensionality of neural signals, resulting in poor generalization performance.

Recently, several studies have successfully reduced the high-dimension neural data into a low-dimension space (two-dimensional or three-dimensional space) for visualization and found potential patterns. Kristan and Calabrese investigated the swimming of leeches and found that single neurons display firing rate oscillations at $\sim 1.5 \mathrm{~Hz}$ [20]. Churchland et al. projected the neural population responses to a two-dimensional space with the jPCA algorithm, and the responses showed rotation trajectories in this rhythm movement [21]. They also recorded electrical signals in monkeys during reaching and found rotary trajectories, even though reaching is not a rhythm movement [21]. Meanwhile, Hao et al. obtained similar results with the Laplace Eigenmaps algorithm [22]. Hence, it is practicable and reasonable to explore whether there is a periodic trajectory for normal and aging subjects at the resting state, and whether these periodic trajectory phenomena are altered with damage to the brain, such as in the pathophysiological processes of Alzheimer's disease $(\mathrm{AD})$.

In fact, dimensionality reduction provides us with an approach to observe the potential patterns of high-dimension neural signals. To date, there are few studies on visualization of fMRI data. In this study, we tried to explore the intrinsic dynamics of brain systems by studying potential patterns in rs-fMRI data between the young and the elderly. The jPCA algorithm was applied to reduce the high-dimension BOLD signal into a two-dimensional space for visualization of its dynamic trajectory. Subsequently, we quantitatively analyzed the trajectory to investigate its dynamic patterns and the working mechanism of the brain system. Finally, the same experiments were applied to the rs-fMRI data of $\mathrm{AD}$ and the normal controls (NC) to further investigate changes in the patterns in the brain in a disordered state.

\section{Materials and Methods}

\subsection{Subjects}

2.1.1. Cohort I. Twenty young adults ( 8 males; mean age: 22.6 years; range: $19-30$ years) and twenty elderly adults (10 males; mean age: 64.1 years; range: 56-79 years) were recruited from the International Consortium for Brain Mapping (ICBM) dataset (http://fcon_1000.projects.nitrc.org/ fcpClassic/FcpTable.html). We performed the experiments on these two groups consecutively.

2.1.2. Cohort II. Thirty-three AD patients (17 males; mean age: 67.3 years; range: $55-89$ years) and thirty-three normal controls (18 males; mean age: 65.2 years; range: $55-85$ years) were recruited from the Alzheimer's Disease Neuroimaging Initiative (ADNI) dataset (http://adni.loni.usc.edu/).

2.2. Data Preprocessing. The rs-fMRI images of cohort I were obtained on a $3 \mathrm{~T}$ scanner with the following parameters: slice number $=133$ and $\mathrm{TR}=2 \mathrm{~s}$. The first five images were discarded to ensure magnetization equilibrium. The images of cohort II were obtained on a 3T scanner with slice number $=140$ and $\mathrm{TR}=2 \mathrm{~s}$. The first 10 images were discarded.

The images of both cohorts were preprocessed with SPM12 (http://www.fil.ion.ucl.ac.uk/spm/) and DPARSF [23]. First, we corrected all the images in the time domain by slice timing. Second, realignment was applied to remove the artifacts in the BOLD signals. Subjects whose head translation was more than $2 \mathrm{~mm}$ or whose head rotation exceeded $2^{\circ}$ were excluded (subjects in our study had already been screened). Third, all the datasets were normalized to the Montreal Neurological Institute (MNI) template. The images were smoothed with a standard $4 \mathrm{~mm}$ fullwidth-at-half-maximum Gaussian kernel to decrease the effects of spatial noise. Fourth, the datasets were drifted and filtered to four frequency bands, that is, slow 2-slow 5. These frequency bands cover $0.198-0.25$ (slow 2), 0.073-0.198 (slow 3), 0.027-0.073 (slow 4), and $0.01-0.027 \mathrm{~Hz}$ (slow 5), respectively. These bands are widely used in resting state fMRI studies [24, 25]. Next, the covariates, including six head motion parameters, global mean signal, white matter signal, and cerebrospinal fluid signal, were removed. Finally, we divided the whole brain into 90 regions according to the AAL template [26-28] and averaged the BOLD signals of each voxel belonging to the same region to represent the BOLD fluctuation of this region. Thus, the state of the brain at one sampling point can be described by a vector with the length of 90 .

2.3. Data Postprocessing. Given the effects of hysteresis of hemodynamics and the relationship between the current and previous signals $[29,30]$, we added the previous $N(N=20$ in this study) frames of signals to the current feature vector. As a result, the original feature space dimension was expanded from 90 (brain regions) to $(1+N) \times 90$. 
2.4. jPCA Algorithm. The jPCA algorithm was proposed by Churchland et al., and they applied it to analyze the firing rate data in monkeys during reaching [21]. Not only can this algorithm retain the most variance of data while reducing dimensionality, like principal component analysis does, but it can also capture the most strongly rotary tendency of the data. Since our data were obtained at the resting state, which is not a rhythm movement similar to reaching, we also used this algorithm to reduce the high dimensionality of our data. The specific mathematical underpinnings regarding jPCA can be seen in [21]. They are briefly described as follows.

First, traditional principal component analysis (PCA) was applied to the original feature space to obtain dimensions relevant to the dynamical structure. We followed the approach in [21] and retained the top six principals (PCs) to capture both expansion and rotary structures. Then, we used jPCA to capture the most strongly rotary tendency. Specifically, the application of jPCA yielded six coordinate bases (jPCs) that span exactly the same space as the six principals from PCA. That is, if a pattern cannot be described by the top six PCs, it cannot be described by the six jPCs either and vice versa. The top two jPCs capture the most strongly rotary tendency in data; therefore, we further embedded the data into the space spanned by the two jPCs. As a result, the reduced data, on the one hand, retained the most variance (i.e., expansion and rotary structures); on the other hand, this data captured the most strongly rotary tendency.

In the jPCA computation, the data were fit with a timeinvariant dynamic system:

$$
\dot{x}(t)=M_{\text {skew }} x(t),
$$

where $x(t)$ represents the signal features at time $t, \dot{x}(t)$ is the derivative of $x(t)$, and $M_{\text {skew }}$ is a transfer matrix. Since the jPCA captures the rotary tendency, $M_{\text {skew }}$ is an antisymmetric matrix. Thus, the aim of jPCA is to solve the optimal $M_{\text {skew: }}$ :

$$
M^{*}=\underset{M}{\arg \min }\|\dot{X}-X M\| .
$$

\section{Results}

3.1. The Fast Fourier Transform (FFT) for the BOLD Signals of the Cohort I. We performed FFT (the window width was the length of the signal) for the BOLD signals of each ROI and each subject in cohort I. For convenience, we only displayed the hippocampus (AAL ID = 37), as it is one of the regions known to be related to aging and $\mathrm{AD}$, of the first subject in the young group (Figure 1), and the results of other ROIs and subjects are quite similar. The peak frequencies in four bands were below $0.1 \mathrm{~Hz}$, which was consistent with most resting state fMRI studies, which concluded that the intrinsic frequency of resting state fMRI was below $0.1 \mathrm{~Hz}$.

We further identified that there were no significant differences ( $t$-test, $\alpha=0.05)$ in peak frequency among all the brain regions in any frequency band neither within a group nor between groups. The overall mean frequencies of all brain regions and all subjects were $0.0112,0.0843,0.0422$, and $0.0196 \mathrm{~Hz}$ in the four bands, corresponding to periods of $89.29,11.86,23.70$, and $51.02 \mathrm{~s}$, respectively. This verified our hypothesis that the BOLD signal, as the basis of functional connectivity, shows a steady periodicity.

3.2. Dimensionality Reduction with the jPCA. After dimensionality reduction for the feature matrix of each subject with jPCA, as shown in Figure 2 (cubic spline interpolation was performed for all trajectories), the trajectories of all subjects, especially at band slow 4, showed a clockwise cycle. These results are consistent with those for rhythmic motion in [21], indicating that the "resting" state is not a completely static state but a basic state that consists of a low frequency and long duration, which could contribute to periodic introspection and low-frequency basal metabolism.

It is interesting that the rotary trend looks more obvious at band slow 4 and degrades as the frequency increases or decreases. We will demonstrate this observation quantificationally later. The BOLD projections in the elderly subjects can be seen in Figure 3, and very similar results were obtained. Specifically, the trajectories show clockwise cycles and appear more rotary at band slow 4 .

$T$-test, at a significance level of 0.05 , demonstrated that there were no significant differences in the centroids of the trajectories between groups. We further utilized the "direct ellipse fit" to fit each trajectory as an ellipse [31]. The results showed that, in both the young and the elderly groups, the long axes and short axes of each ellipse are quite similar and the cross-term was approximately zero, indicating that the fitted trajectories are approximate circles. The fitted trajectories at band slow 4 are shown in Figure 4.

We subsequently transformed the coordinates of $\mathrm{jPC1}$ and jPC2 to the polar coordinates for all the trajectories in both groups. After FFT, the overall mean peak frequencies of phase angles in the four bands were $0.0113,0.0839,0.0418$, and $0.0198 \mathrm{~Hz}$ in the young participants and $0.0130,0.0815$, 0.0398 , and $0.0188 \mathrm{~Hz}$ in the elderly participants, respectively, which are consistent with those of the BOLD signals. Moreover, no significant difference in peak frequency was found in any band between the young and the elderly participants. As this variable directly reflects the rotary frequency of the trajectory, we therefore regarded its reciprocal as the rotary period of the trajectory. Thus, the rotary period between the young and the elderly participants shows no significant difference.

Combining the analyses of the time and frequency domains, we could infer that the trajectory of resting state BOLD signals after dimensionality reduction is approximately circular in different frequency bands.

3.3. Validation. Two criteria must be satisfied if a twodimensional (e.g., $X$ and $Y$ ) signal is a circle. First, the phase difference between $X$ and $Y$ should be stable. Second, the frequencies in $X$ and $Y$ should be stable. We validated the reduced signals in our study; the phase difference between $\mathrm{jPC} 1$ and $\mathrm{jPC} 2$ is approximately $\pi / 2$ (take the first subject of the young at band slow 4, e.g., in Figure 5). We performed FFT for the two jPCs as well, and the peak frequencies were $0.0407 \mathrm{~Hz}$ in $\mathrm{jPCl}$ and $0.0413 \mathrm{~Hz}$ in $\mathrm{jPC}$. The BOLD signal therefore satisfied the two criteria. On the other hand, the 


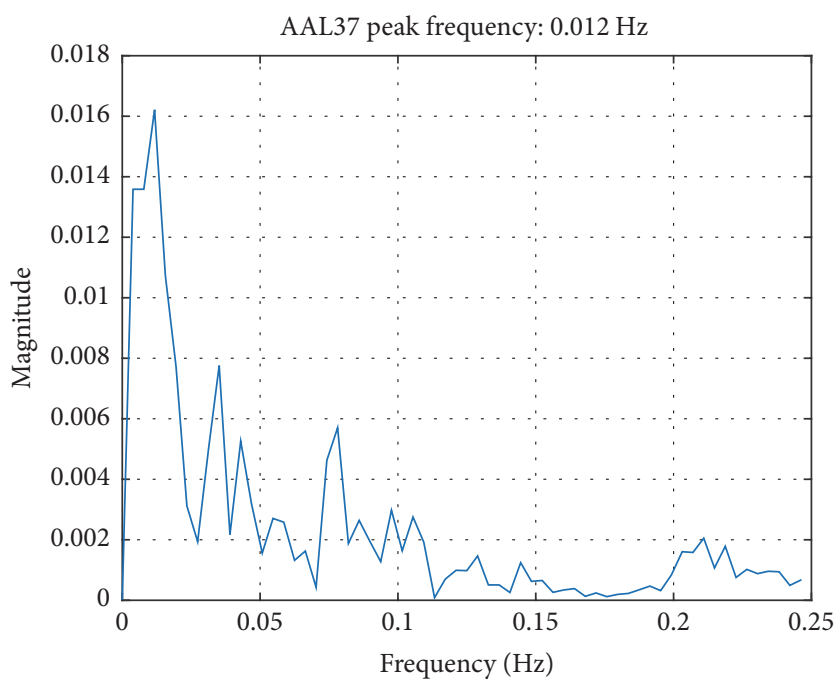

(a) Band slow 2

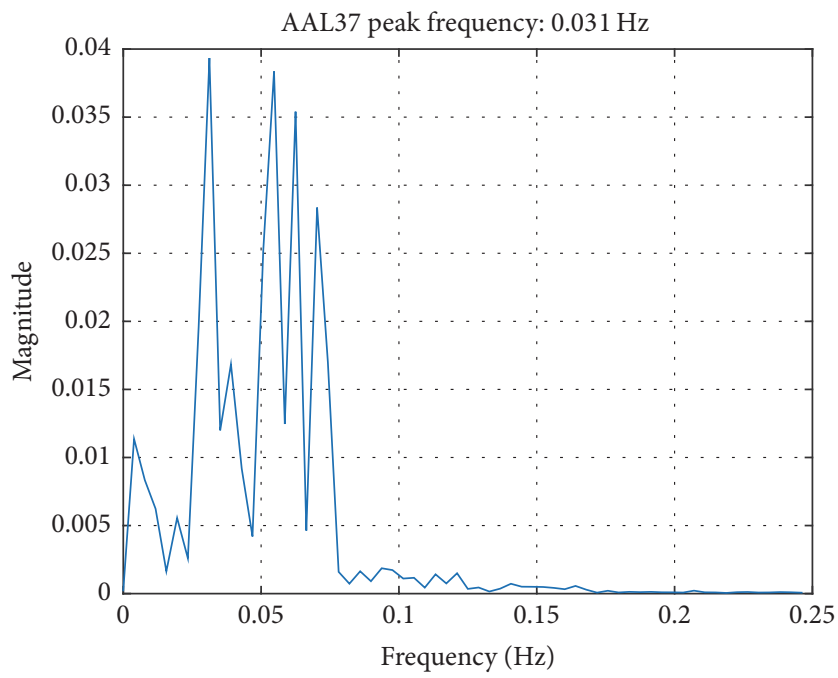

(c) Band slow 4

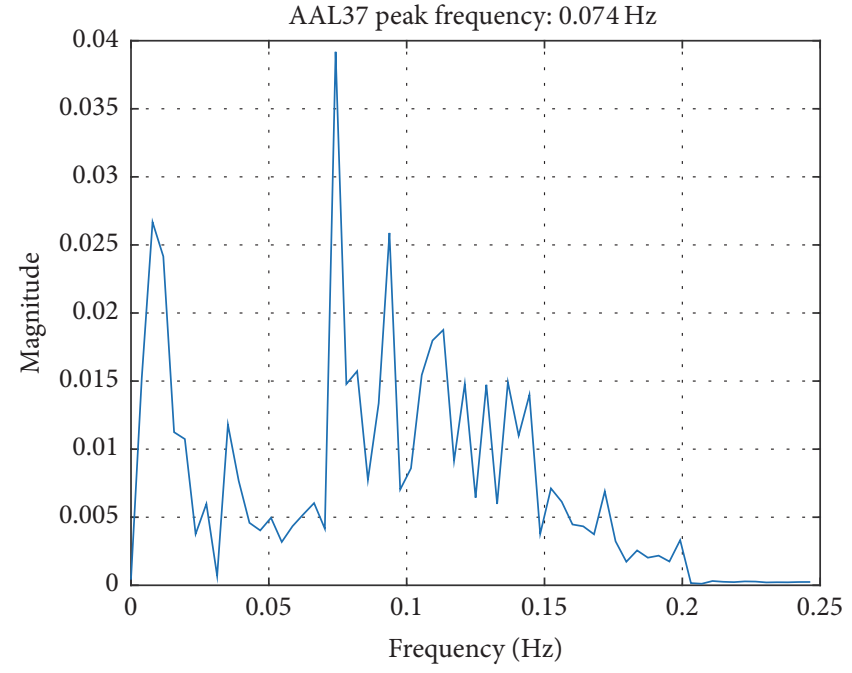

(b) Band slow 3

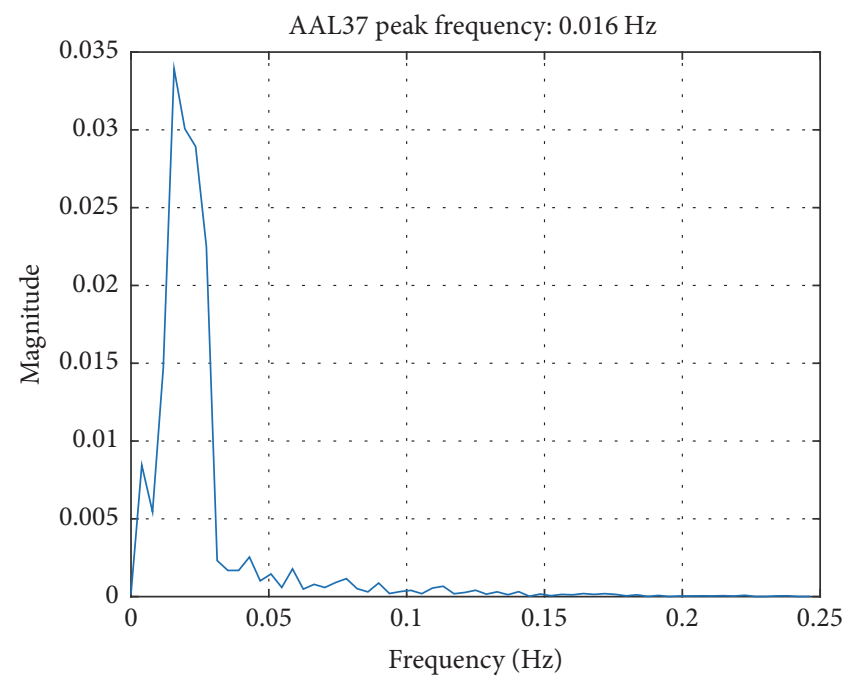

(d) Band slow 5

FIGURE 1: FFT for BOLD signals (for convenience, only the hippocampus of the first subject in each group is shown; the results of other ROIs and subjects are quite similar).

peak frequency in the reduced signals is consistent with that in the raw BOLD signals, indicating that the reduced signals well captured the frequency features in raw signals.

In the jPCA algorithm, the data were fitted with

$$
\dot{x}(t)=M_{\text {skew }} x(t)
$$

where $x(t)$ is the neural state for time $t$ and $M_{\text {skew }}$ is a skewsymmetric matrix when capturing rotational dynamics. The unconstrained $M$ therefore provides the best fit of all possible matrices. We evaluated the fits under the two conditions and found that $M_{\text {skew }}$ was nearly as good as the fit by $M$ at band slow 4 ( $M: R^{2}=0.97 ; M_{\text {skew }}: R^{2}=0.83$ for the young; $R^{2}=0.81$ for the old). This result is consistent with that in [21] and much higher than that obtained with velocitytuned and complex-kinematic models (which can be seen as motor executive signals), indicating that the trajectory can be described with cycle rotation.

The coefficients of determination in the four bands were $0.41,0.71,0.83$, and 0.70 in the young group and $0.45,0.72$, 0.81 , and 0.65 in the old group, respectively. That is, the rotary performance is best at slow 4 and degrades with an increase or decrease in the frequency.

We calculated the angle between $x$ and its derivative. This angle reflects the rotation strength of the trajectory, and the rotation is strong when the angle is close to $\pi / 2$. As shown in Figure 6, the peak angle is around $\pi / 2$ at slow 4 and much higher than other motor executive signals [21].

The results mentioned above indicate that (1) the trajectory rotates and (2) the neural signals are homologous. That is, the BOLD and neural firing rate signals are both derived from the neural system. Their dynamics are quite similar 


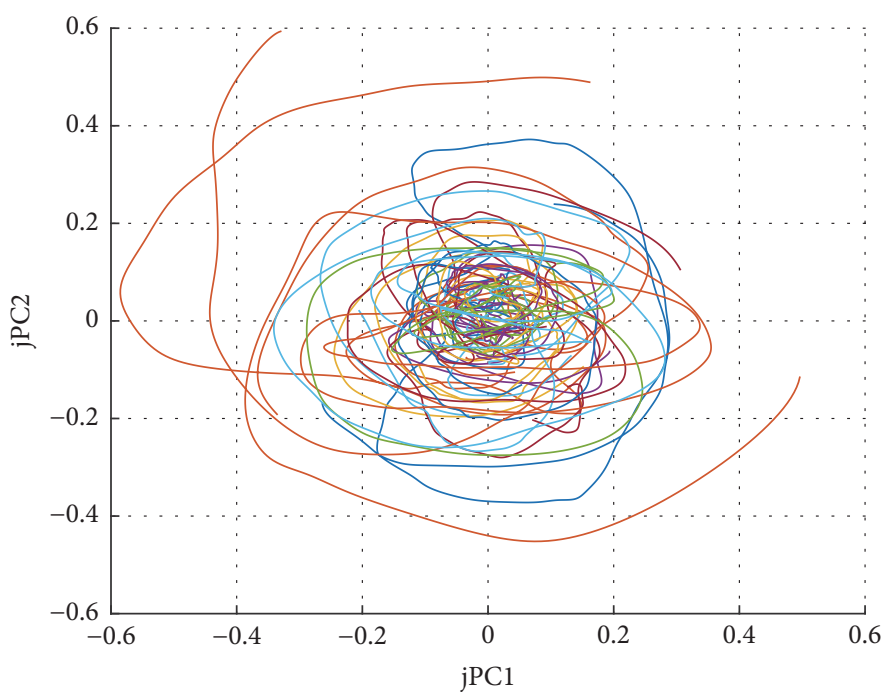

(a) Slow $2(0.198-0.25 \mathrm{~Hz})$

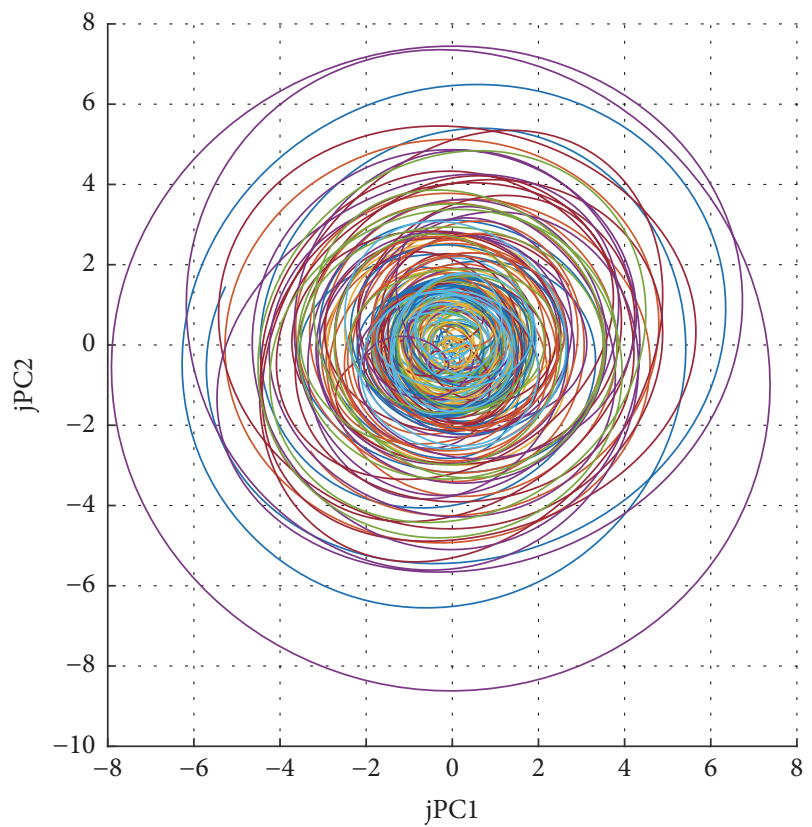

(c) Slow $4(0.027-0.073 \mathrm{~Hz})$

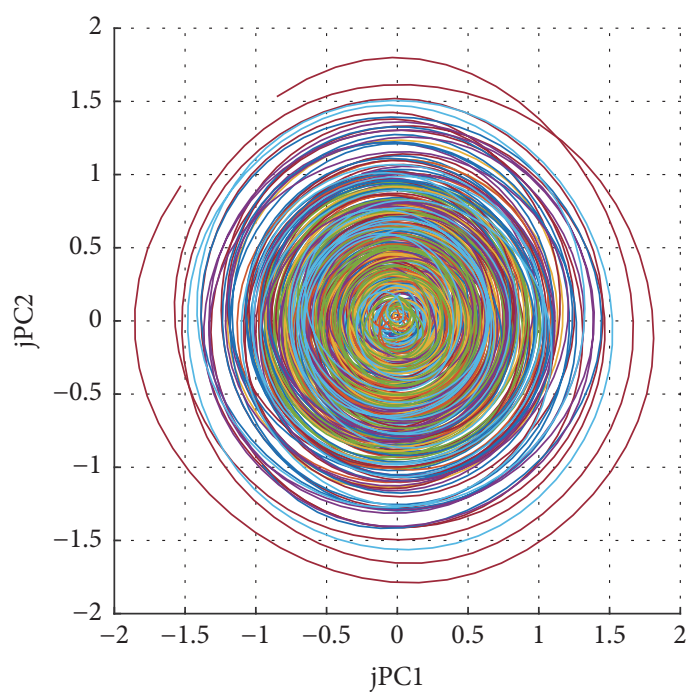

(b) Slow $3(0.073-0.198 \mathrm{~Hz})$

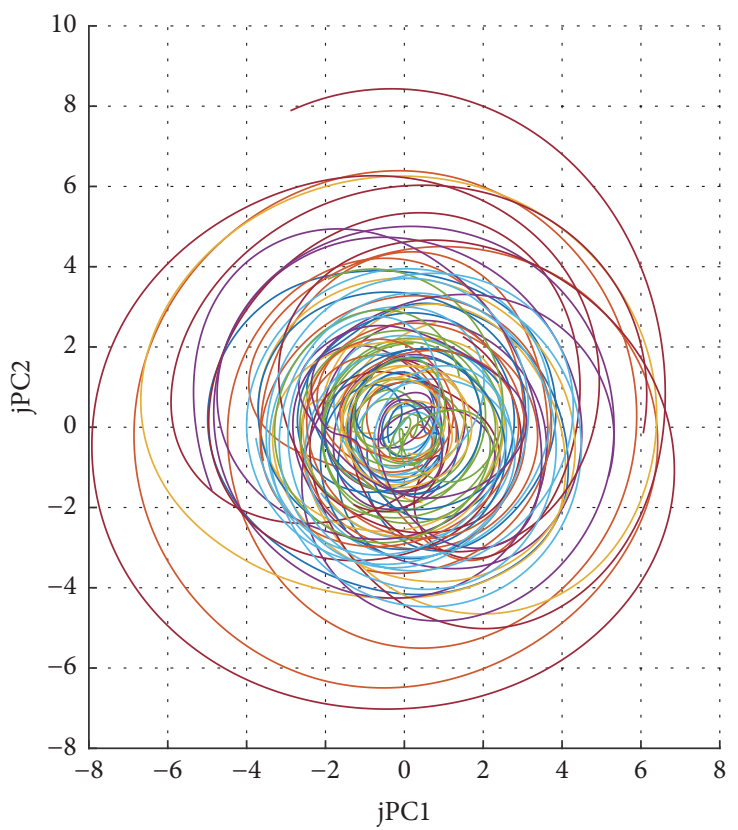

(d) Slow $5(0.01-0.027 \mathrm{~Hz})$

FIGURE 2: BOLD trajectories after projection with jPCA (one color represents one person of the young group; all trajectories were obtained with cubic spline interpolation).

and different from those of the motor executive signals. Churchland et al. found that electromyograms (EMGs) did not show consistent rotations themselves, but EMGs can be generated by low-frequency components of two rotations, indicating that the upper signals (secondary signals) do not rotate themselves but consist of lower neural signals with rotary dynamics [21]. However, in our study, the BOLD signals are secondary signals but exhibit rotary dynamics as well. Thus, we considered that the original conclusion could be adjusted as follows: the neural signals contain rotary patterns, and the motor executive signals consist of neural signals.
Meanwhile, through statistical tests, we found no differences in any result between the young and elderly participants. Specifically speaking, the trajectories in both groups rotate with frequencies that are consistent with the peak frequencies in the BOLD signals. The biggest coefficient of determination was noted at slow 4 . We therefore inferred that the rotary pattern may be relative to the basal metabolism and may be present throughout an individual's life regardless of age. This rotary pattern does not change with normal aging from the global perspective. In the next section, we investigated the rotary pattern in Alzheimer's disease to find whether it changes in a disordered brain. 


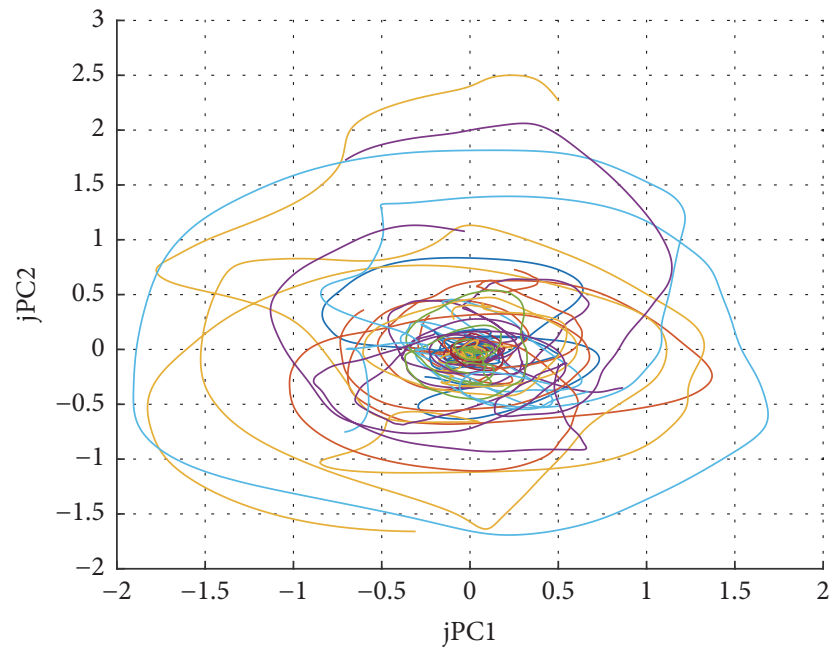

(a) Slow $2(0.198-0.25 \mathrm{~Hz})$

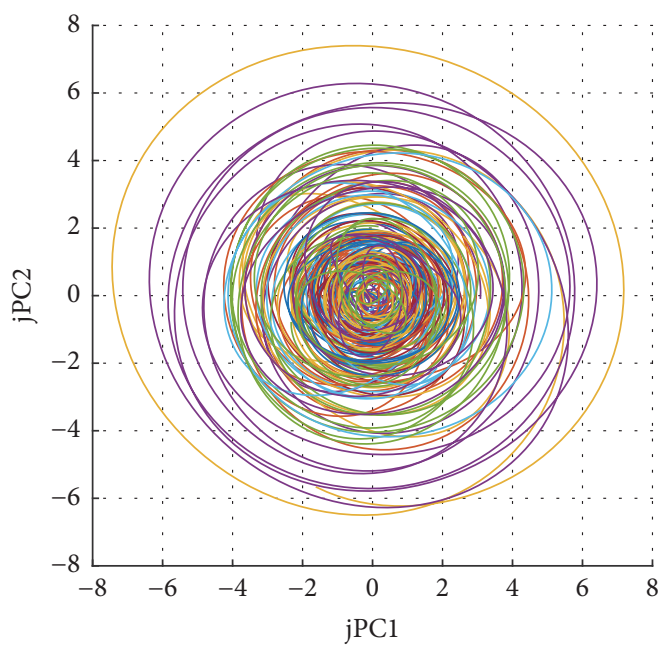

(c) Slow $4(0.027-0.073 \mathrm{~Hz})$

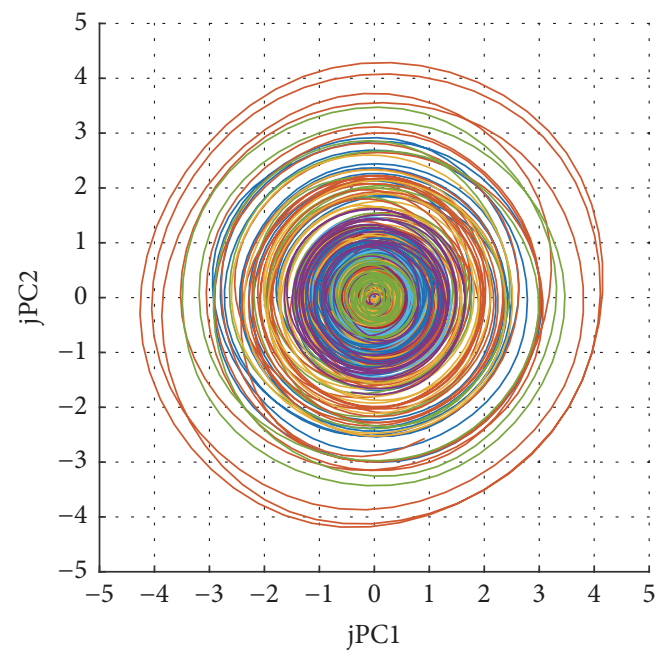

(b) Slow $3(0.073-0.198 \mathrm{~Hz})$

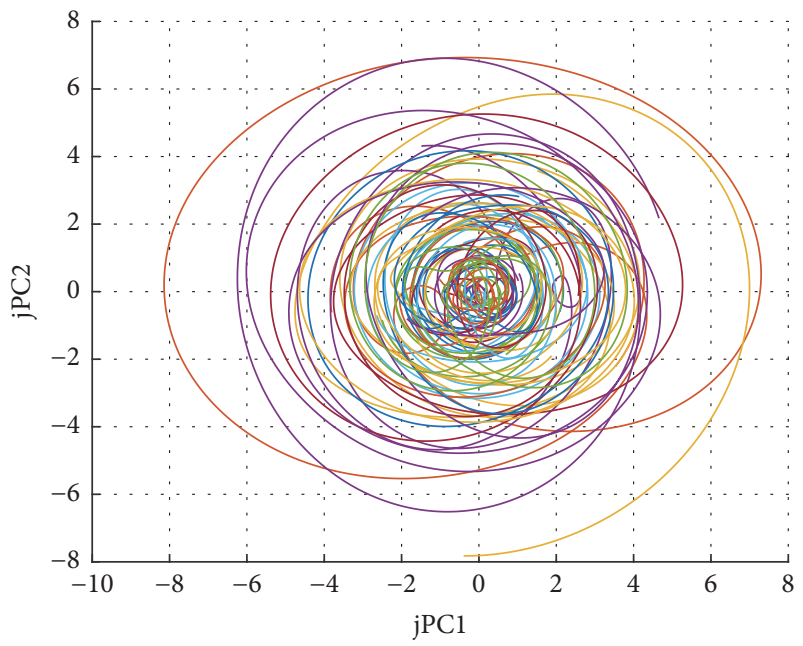

(d) Slow $5(0.01-0.027 \mathrm{~Hz})$

FIGURE 3: BOLD trajectories after projection with jPCA (one color represents one person of the elderly group; all trajectories were obtained with cubic spline interpolation).

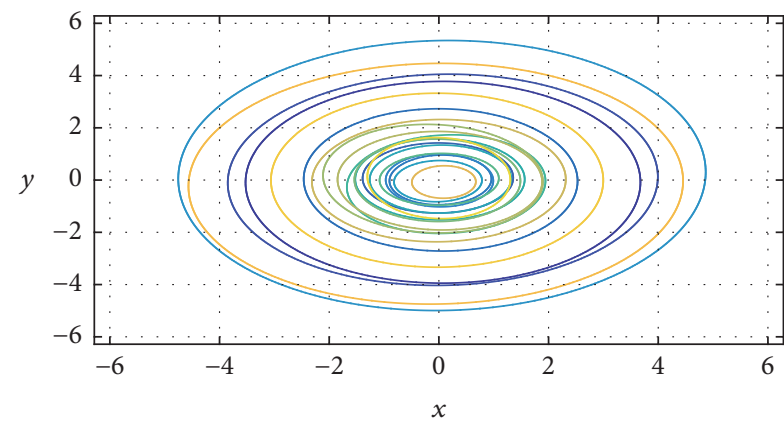

(a) Ellipse fits of the young

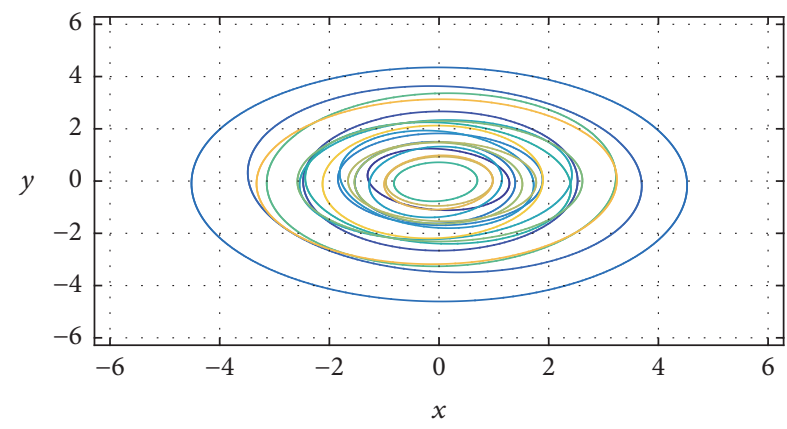

(b) Ellipse fits of the old

FIGURE 4: Direct ellipse fits for trajectories of (a) the young and (b) the elderly participants at band slow 4. 


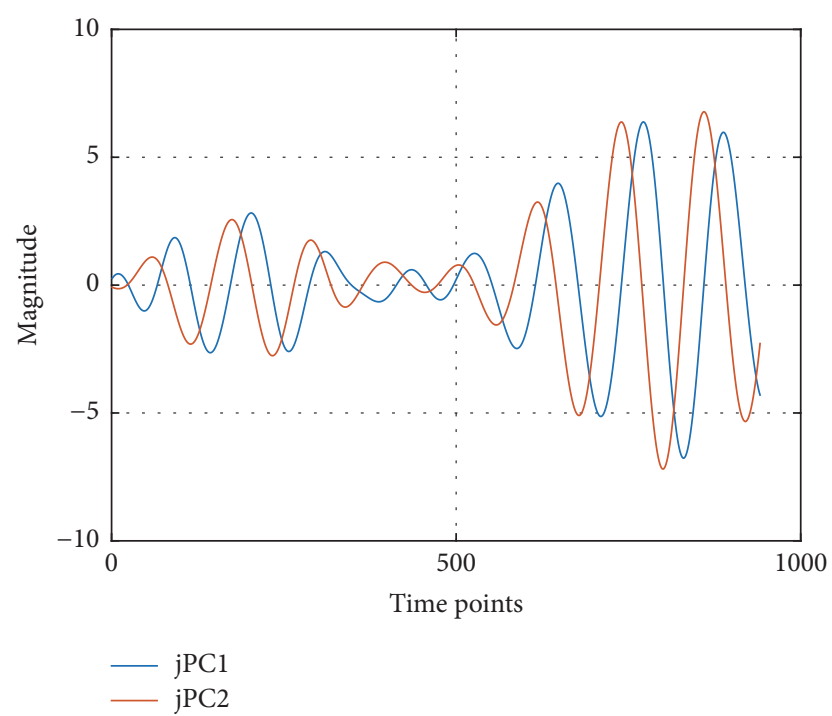

FIGURE 5: The projections (of the first subject in the young group) onto the two-dimensional jPCs versus time.

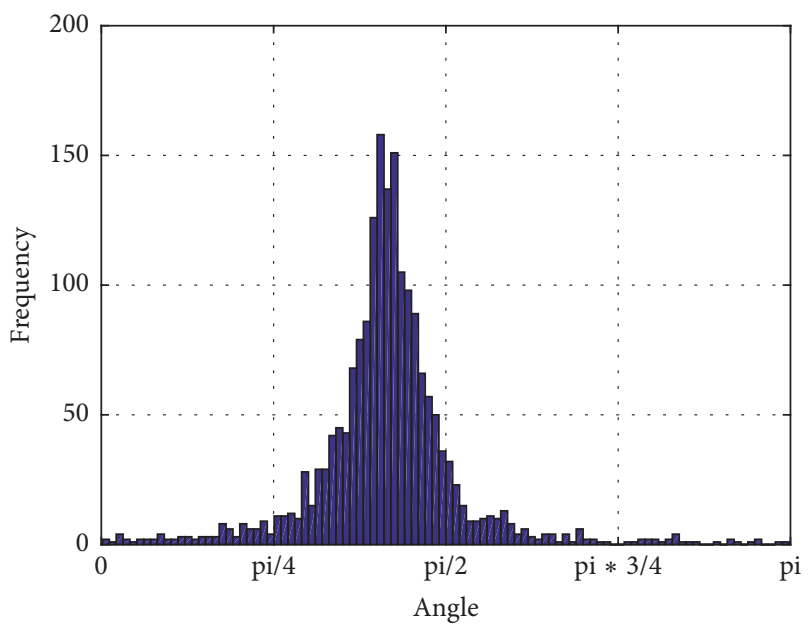

FIgURE 6: Angle distribution. The angle between $x$ and its derivative of the first subject in the young group at band slow 4 .

3.4. Experimental Results for the AD and NC Groups. The rotary performance in the $\mathrm{AD}$ and $\mathrm{NC}$ groups was similar to that in the young and the elderly groups. We have briefly summarized the results of the AD and NC groups. The trajectories of both groups rotate at a long period at different bands (e.g., slow 4 in Figure 7), and the period (or frequency) is consistent with that of the BOLD signals. No significant intergroup difference was noted in the centroid of the trajectory. For both the $\mathrm{AD}$ and $\mathrm{NC}$ groups, the biggest coefficient of determination for skew-symmetricmatrix fitting is at band slow $4\left(R^{2}=0.83\right.$ for the $\mathrm{AD}$, and 0.82 for the NC), and the coefficient degrades as the frequency increases or decreases.

The exciting aspect about these findings is that the rotary period between the AD and NC groups shows significant difference $(t$-test, $P$ value $<0.05)$ at band slow 4 . The rotary period in the $\mathrm{AD}$ group is $24.45 \mathrm{~s}(0.0409 \mathrm{~Hz})$ and that in the NC group is $22.37 \mathrm{~s}(0.0447 \mathrm{~Hz})$. Thus, the rotary pattern slows down in the $\mathrm{AD}$, which is known as a brain disordered state.

Combining these findings with the results in the young and elderly groups, we could infer that the rotary pattern is associated with normal aging regardless of age but changes when the brain shows some kind of disorder. Thus, the rotary period at band slow 4 may be a physiological marker for $\mathrm{AD}$, and studies of this frequency band may be useful for understanding the potential pathophysiology of $\mathrm{AD}$ and ultimately help in characterization and auxiliary diagnosis of AD.

\section{Discussion}

Researchers usually assume that neural signals reflect physical and mental movements [2-5]; therefore, periodic activities are expected to generate periodic fluctuations. Recent studies support this assumption and have found periodic neural patterns in many rhythm movements [20, 21]. Furthermore, some studies found rotary patterns in monkeys during reaching, which is not a rhythm movement [21, 22]. Churchland et al. indicated that the EMG signals were generated by low-frequency components of two rotations; that is, upper or secondary signals consist of basic neural signals. This is the reason why the EMGs cannot rotate themselves but the firing rate signals can [21].

In our study, we investigated the neural trajectories in young and elderly adults as well as in $\mathrm{AD}$ patients at four frequency bands (slow 2-slow 5) in the resting state based on BOLD signals, expanding the findings of previous studies. First, the resting state is not a strict rhythm movement. Although low-frequency basal metabolism and periodic introspection constitute the main components of "resting activities," a mass of mind drifts and responses to undetermined environments influence the neural activities. Rotary trajectories were found in the resting state, supporting the conclusion that neural activities showed periodic patterns during nonrhythmic movements. Second, BOLD signals are upper secondary signals (rather than basic neural signals) but showed rotary trajectories as well. This can imply that signals from neural system show rotary trajectories, and the neurons in motor executive systems only receive part of the control signals from the neural system. The trajectories of signals in the motor executive systems (e.g., those based on velocitytuned and complex-kinematic models and EMG) therefore cannot rotate themselves.

In comparisons between groups, one interesting result is that there were no significant differences in the centroids of trajectories between young and elderly adults but the rotary pattern in the $\mathrm{AD}$ patients slowed down at band slow 4. One possible explanation for these results is that alterations of the rotary pattern in normal controls are still undetectable due to limitations of the sample size. Another explanation is the possibility that the rotary pattern occurs with normal aging regardless of age, but changes may appear at the band slow 4 when the brain is of some kind of disorder such as in $\mathrm{AD}$. A previous study reported that slow 4 bands 


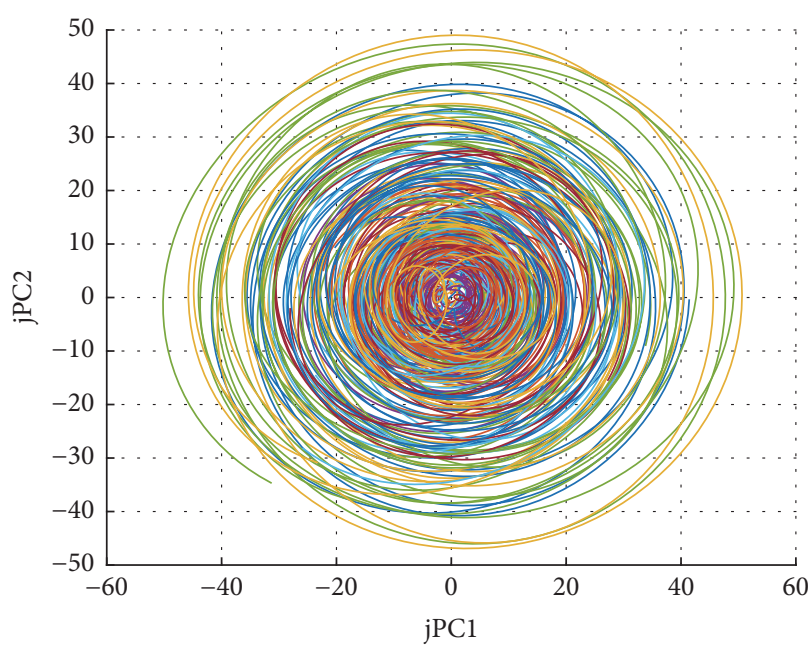

(a) Trajectories of Alzheimer's disease

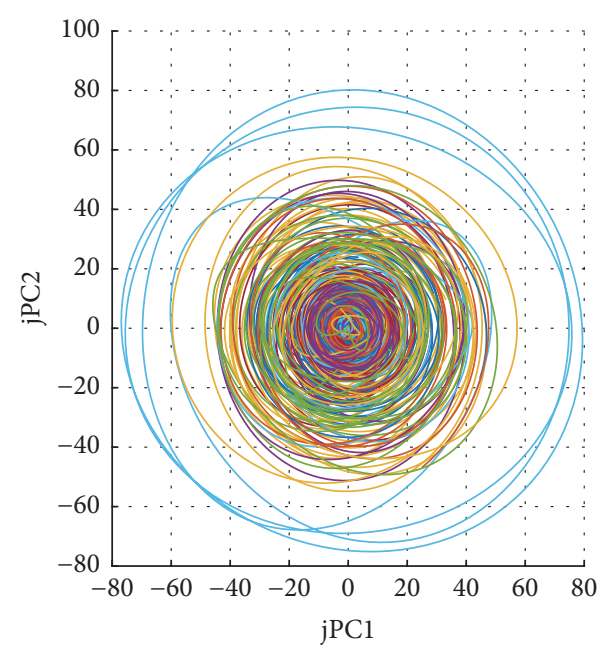

(b) Trajectories of the normal controls

FIGURE 7: Trajectories at band slow 4 of (a) the AD group and (b) the NC group (one color represents one person; all trajectories were obtained with cubic spline interpolation).

mainly reflect gray matter signals in the brain and showed the most robust findings in the basal ganglia in spontaneous electrophysiological recordings [25]. Abnormalities in intrinsic brain activity in this frequency band, identified using resting state functional MRI, were also reported in other ADrelated spectrum disorders such as amnestic mild cognitive impairment and $\mathrm{ApoE} \varepsilon 4$ allele carriers, indicating the need for meaningful further research on the characterization of slow 4 bands [32-34]. Combined with our findings, the rotary period at band slow 4 may be a useful physiological marker for diseases with $\mathrm{AD}$-related brain damage. In our study, we recorded the BOLD signals from the whole brain rather than specific electrodes in a few regions. Thus, the trajectories can reflect the activities of the whole brain without bias, indicating that the "resting state" is not an entirely static state but a basic state with a low frequency and long period.

Reduction of the dimensionality of neural signals not only allowed identification of potential patterns but also helped remove the noise for statistical analyses. This is of profound significance in coding and decoding neural signals.

\section{Conclusions}

We used the jPCA algorithm to reduce the dimensionality of BOLD signals and found rotary trajectories during the resting state. The results indicated that the "resting state" is a basic state, showing an inherent dynamic pattern with a low frequency and long period, and expand the original conclusion to that neural signals can rotate themselves and that motor executive signals consist of neural signals. Moreover, alterations in the rotary trajectories at the slow 4 frequency band $(0.027-0.073 \mathrm{~Hz})$ were found in patients with Alzheimer's disease (AD), which may be useful for understanding the potential pathophysiology of $\mathrm{AD}$ and ultimately facilitate characterization and auxiliary diagnosis of $\mathrm{AD}$.

\section{Disclosure}

Data used in preparation of this article were obtained from the Alzheimer's Disease Neuroimaging Initiative (ADNI) database (https://adni.loni.usc.edu). As such, the investigators within the ADNI contributed to the design and implementation of ADNI and/or provided data but did not participate in analysis or writing of this report. A complete list of ADNI investigators can be found at http://adni.loni.usc.edu/wp-content/uploads/how_to_apply/ ADNI_Acknowledgement_List.pdf.

\section{Conflicts of Interest}

The authors of this study state that there are no conflicts of interest to disclose.

\section{Acknowledgments}

This work was supported by the National Natural Science Foundation of China $(61473131,60905024)$ and 973 Project of China (2013CB329506). Data collection and sharing for this project was funded by the Alzheimer's Disease Neuroimaging Initiative (ADNI) (National Institutes of Health, Grant no. U01 AG024904) and DOD ADNI (Department of Defense Award no. W81XWH-12-2-0012). ADNI is funded by the National Institute on Aging, the National Institute of Biomedical Imaging and Bioengineering, and generous contributions from the following: AbbVie, Alzheimer's Association; Alzheimer's Drug Discovery Foundation; Araclon Biotech; BioClinica, Inc.; Biogen; Bristol-Myers Squibb Company; CereSpir, Inc.; Cogstate; Eisai Inc.; Elan Pharmaceuticals, Inc.; Eli Lilly and Company; EuroImmun; F. HoffmannLa Roche Ltd. and its affiliated company Genentech, Inc.; Fujirebio; GE Healthcare; IXICO, Ltd.; Janssen Alzheimer Immunotherapy Research \& Development, LLC; Johnson 
\& Johnson Pharmaceutical Research \& Development, LLC; Lumosity; Lundbeck; Merck \& Co., Inc.; Meso Scale Diagnostics, LLC; NeuroRx Research; Neurotrack Technologies; Novartis Pharmaceuticals Corporation; Pfizer, Inc.; Piramal Imaging; Servier; Takeda Pharmaceutical Company; and Transition Therapeutics. The Canadian Institutes of Health Research is providing funds to support ADNI clinical sites in Canada. Private sector contributions are facilitated by the Foundation for the National Institutes of Health (https://www.fnih.org). The grantee organization is the Northern California Institute for Research and Education, and the study is coordinated by the Alzheimer's Therapeutic Research Institute at the University of Southern California. ADNI data are disseminated by the Laboratory for Neuro Imaging at the University of Southern California.

\section{References}

[1] R. L. Carhart-Harris and K. J. Friston, "The default-mode, ego-functions and free-energy: A neurobiological account of Freudian ideas," Brain, vol. 133, no. 4, pp. 1265-1283, 2010.

[2] E. V. Evarts, "Relation of pyramidal tract activity to force exerted during voluntary movement.," Journal of Neurophysiology, vol. 31, no. 1, pp. 14-27, 1968.

[3] J. F. Kalaska, "From intention to action: Motor cortex and the control of reaching movements," Advances in Experimental Medicine and Biology, vol. 629, pp. 139-178, 2009.

[4] E. Todorov, "Direct cortical control of muscle activation in voluntary arm movements: A model," Nature Neuroscience, vol. 3, no. 4, pp. 391-398, 2000.

[5] T. N. Aflalo and M. S. Graziano, "Relationship between unconstrained arm movements and single-neuron firing in the macaque motor cortex," The Journal of Neuroscience, vol. 27, no. 11, pp. 2760-2780, 2007.

[6] A. P. Georgopoulos, A. B. Schwartz, and R. E. Kettner, "Neuronal population coding on movement direction," Science, vol. 233, no. 4771, pp. 1416-1419, 1986.

[7] C. Vidaurre, J. Pascual, A. Ramos-Murguialday et al., "Neuromuscular electrical stimulation induced brain patterns to decode motor imagery," Clinical Neurophysiology, vol. 124, no. 9, pp. 1824-1834, 2013.

[8] L.-L. Zeng, Y. Liao, Z. Zhou et al., "Default network connectivity decodes brain states with simulated microgravity," Cognitive Neurodynamics, vol. 10, no. 2, pp. 113-120, 2016.

[9] N. K. Logothetis, "What we can do and what we cannot do with fMRI," Nature, vol. 453, no. 7197, pp. 869-878, 2008.

[10] M. Fukunaga, S. G. Horovitz, P. van Gelderen et al., "Largeamplitude, spatially correlated fluctuations in BOLD fMRI signals during extended rest and early sleep stages," Magnetic Resonance Imaging, vol. 24, no. 8, pp. 979-992, 2006.

[11] C. Yeh, Y. Tseng, Y. Lin, S. Tsai, and T. Huang, "RestingState Functional Magnetic Resonance Imaging: The Impact of Regression Analysis," Journal of Neurogenetics, vol. 25, pp. 117123, 2015.

[12] G. Buzsaki, K. Kaila, and M. Raichle, "Inhibition and brain work," Neuron, vol. 56, pp. 771-783, 2007.

[13] B. Biswal, F. Z. Yetkin, V. M. Haughton, and J. S. Hyde, "Functional connectivity in the motor cortex of resting human brain using echo-planar MRI," Magnetic Resonance in Medicine, vol. 34, no. 4, pp. 537-541, 1995.
[14] M. J. Lowe, B. J. Mock, and J. A. Sorenson, "Functional connectivity in single and multislice echoplanar imaging using resting-state fluctuations," NeuroImage, vol. 7, no. 2, pp. 119-132, 1998.

[15] D. Cordes, "Mapping Functionally Related Regions of Brain with Functional Connectivity MR Imaging," American Journal of Neuroradiology, vol. 21, pp. 1636-1644, 2000.

[16] M. Razavi, B. Eaton, S. Paradiso, M. Mina, A. G. Hudetz, and L. Bolinger, "Source of low-frequency fluctuations in functional MRI signal," Journal of Magnetic Resonance Imaging, vol. 27, no. 4, pp. 891-897, 2008.

[17] B. Biswal, E. A. DeYoe, and J. S. Hyde, "Reduction of physiological fluctuations in fMRI using digital filters," Magnetic Resonance in Medicine, vol. 35, no. 1, pp. 107-113, 1996.

[18] M. S. Dagli, J. E. Ingeholm, and J. V. Haxby, "Localization of cardiac-induced signal change in fMRI," NeuroImage, vol. 9, no. 4, pp. 407-415, 1999.

[19] P. Fransson, "Spontaneous low-frequency BOLD signal fluctuations: an fMRI investigation of the resting-state default mode of brain function hypothesis," Human Brain Mapping, vol. 26, no. 1, pp. 15-29, 2005.

[20] W. J. Kristan and R. L. Calabrese, "Rhythmic Swimming Activity in Neurones of the Isolated Nerve Cord of the Leech," Journal of Experimental Biology, vol. 65, pp. 643-668, 1976.

[21] M. M. Churchland et al., Neural Population Dynamics during Reaching, Nature, 2012.

[22] Y. Hao et al., "Distinct neural patterns enable grasp types decoding in monkey dorsal premotor cortex," Journal of Neural Engineering, vol. 11, no. 6, 2014.

[23] Y. Chao-Gan and Z. Yu-Feng, "DPARSF: A MATLAB Toolbox for "Pipeline" Data Analysis of Resting-State fMRI," Frontiers in Systems Neuroscience, vol. 4, no. 13, 2010.

[24] M. Penttonen and B. György, "Natural logarithmic relationship between brain oscillators," Thalamus \& Related Systems, vol. 2, no. 2, pp. 145-152, 2004.

[25] X. N. Zuo, A. di Martino, C. Kelly et al., "The oscillating brain: complex and reliable," NeuroImage, vol. 49, no. 2, pp. 1432-1445, 2010.

[26] W. Li, Y. Huang, Y. Li, and X. Chen, "Brain network evolution after stroke based on computational experiments," PLoS One, vol. 8, Article ID e82845, 2013.

[27] R. Sala-Llonch, C. Junqué, E. M. Arenaza-Urquijo et al., "Changes in whole-brain functional networks and memory performance in aging," Neurobiology of Aging, vol. 35, no. 10, pp. 2193-2202, 2014.

[28] N. Tzourio-Mazoyer, B. Landeau, D. Papathanassiou et al., "Automated anatomical labeling of activations in SPM using a macroscopic anatomical parcellation of the MNI MRI singlesubject brain," NeuroImage, vol. 15, no. 1, pp. 273-289, 2002.

[29] R. L. Buckner, "Event-related fMRI and the hemodynamic response," Human Brain Mapping, vol. 6, no. 5-6, pp. 373-377, 1998.

[30] W. Li, Y. Li, C. Hu, X. Chen, and H. Dai, "Point process analysis in brain networks of patients with diabetes," Neurocomputing, vol. 145, pp. 182-189, 2014.

[31] A. Fitzgibbon, M. Pilu, and R. B. Fisher, "Direct least square fitting of ellipses," Pattern Analysis and Machine Intelligence, vol. 21, pp. 476-480, 1999.

[32] Y. Han, J. Wang, and Z. Zhao, "Frequency-dependent changes in the amplitude of low-frequency fluctuations in amnestic mild cognitive impairment: a resting-state fMRI study," NeuroImage, vol. 55, no. 1, pp. 287-295, 2011. 
[33] J. Wang, X. Zuo, Z. Dai et al., "Disrupted functional brain connectome in individuals at risk for Alzheimer's disease," Biological Psychiatry, vol. 73, no. 5, pp. 472-481, 2013.

[34] L. Ying et al., "Frequency Specific Effects of ApoE $\varepsilon 4$ Allele on Resting-State Networks in Nondemented Elders," BioMed Research International, vol. 2017, Article ID 9823501, pp. 1-8, 2017. 


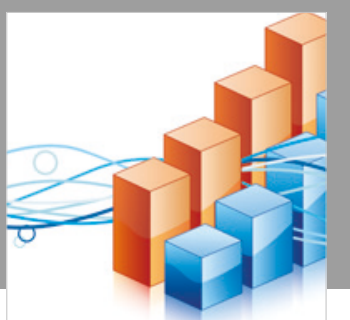

Advances in

Operations Research

\section{- $=$}
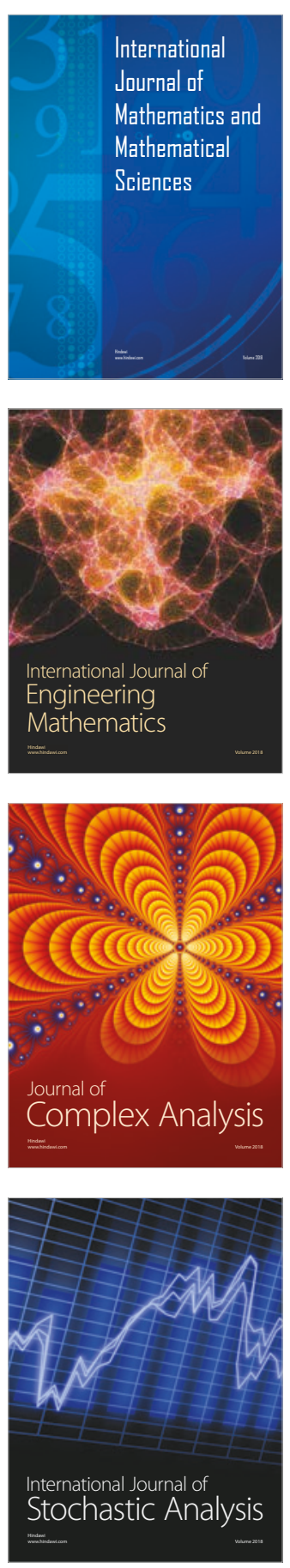

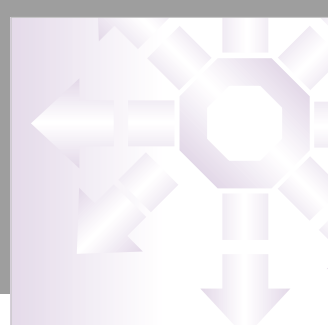

Advances in

Decision Sciences
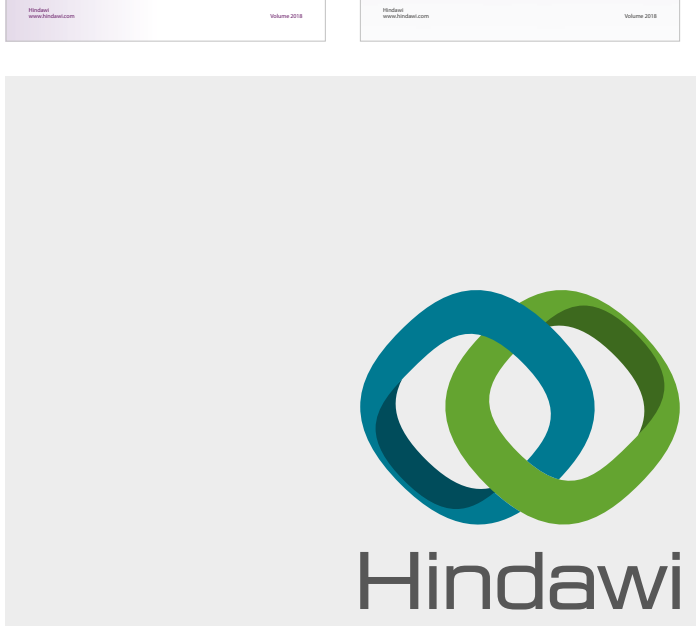

Submit your manuscripts at

www.hindawi.com

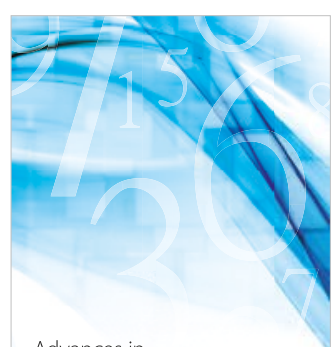

Advances in
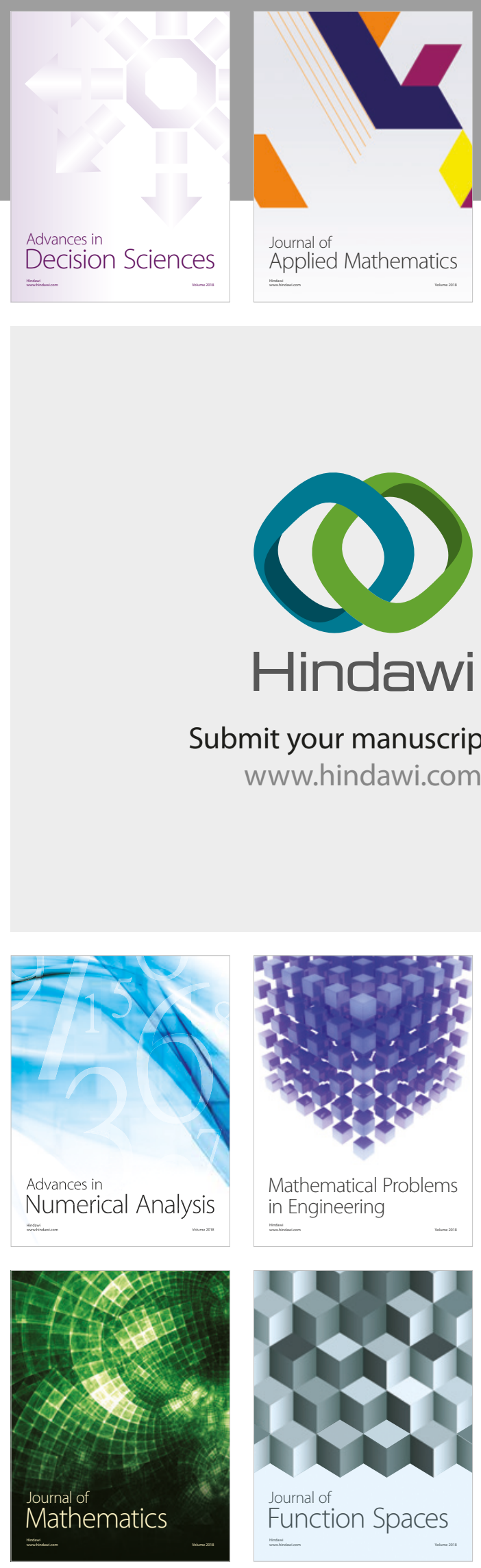

Journal of

Applied Mathematics

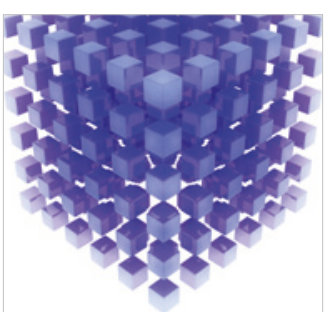

Mathematical Problems in Engineering

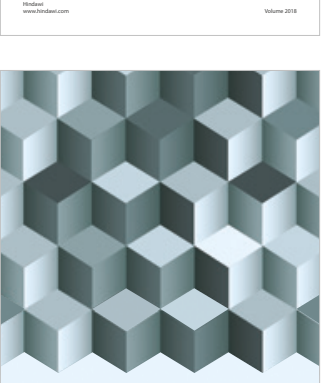

Journal of

Function Spaces

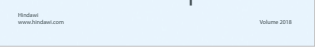

The Scientific

World Journal
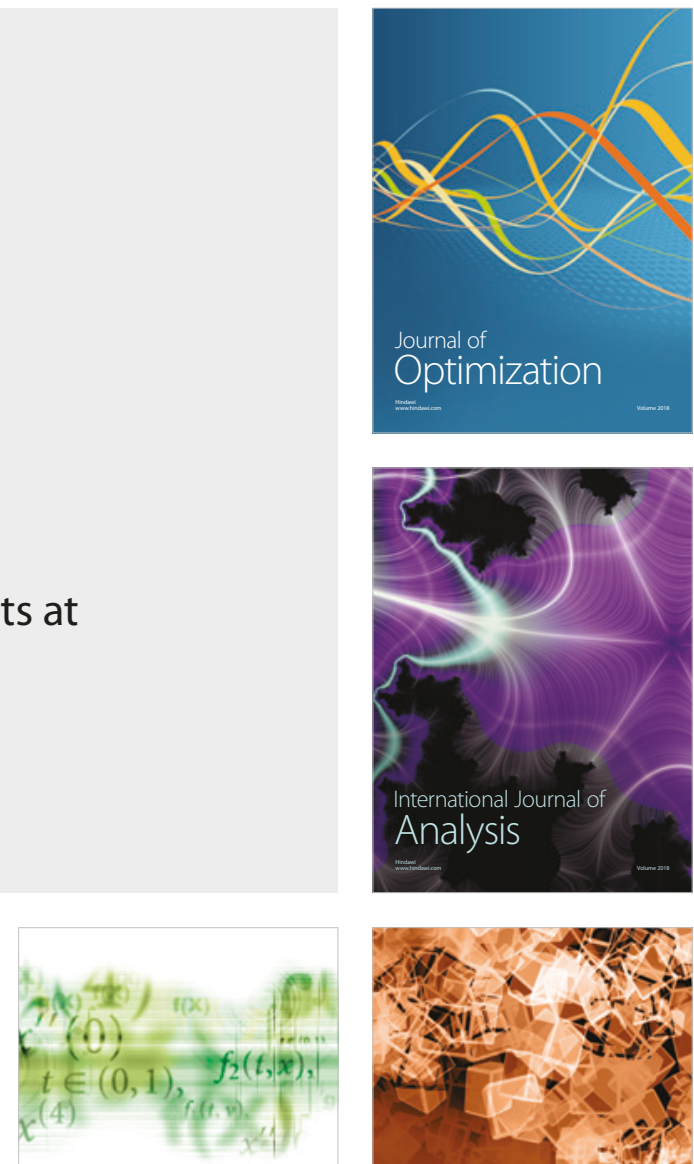

International Journal of

Differential Equations
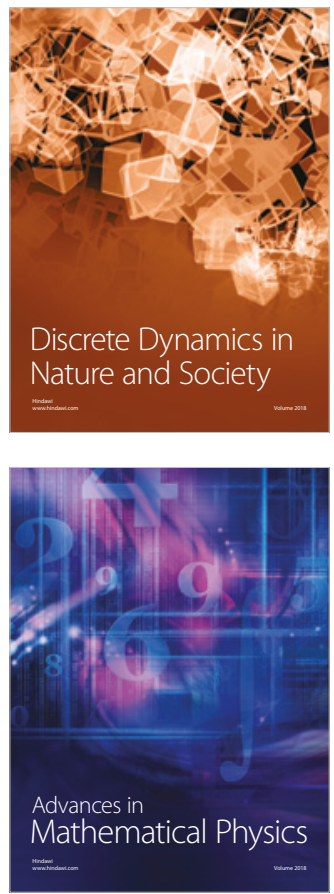

Abstract and Applied Analysis

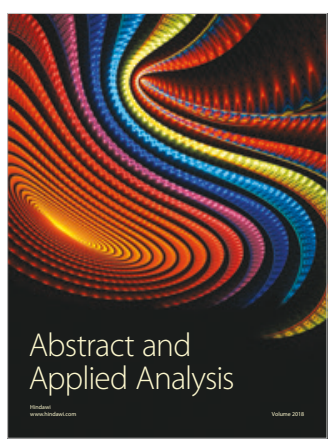

Probability and Statistics

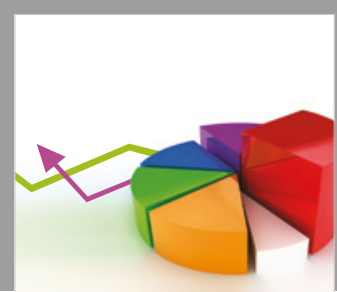

$$
\text { . }
$$

